\title{
Harmonic and Monogenic Potentials in Euclidean Halfspace
}

\author{
F. Brackx, H. De Bie and H. De Schepper \\ Clifford Research Group, Department of Mathematical Analysis, Faculty of Engineering and Architecture, \\ Ghent University, Belgium
}

\begin{abstract}
In the framework of Clifford analysis a chain of harmonic and monogenic potentials is constructed in the upper half of Euclidean space $\mathbb{R}^{m+1}$. Their distributional limits at the boundary are computed, obtaining in this way well-known distributions in $\mathbb{R}^{m}$ such as the Dirac distribution, the Hilbert kernel, the square root of the negative Laplace operator, and the like. It is shown how each of those potentials may be recovered from an adjacent kernel in the chain by an appropriate convolution with such a distributional limit.
\end{abstract}

Keywords: potential theory, Clifford analysis

PACS: 02.30.-f (MSC 30G35)

\section{INTRODUCTION}

In the complex plane the notion of holomorphic potential or holomorphic primitive is linked to the complex derivative $\frac{d}{d z}$. When restricting to the upper half plane, the logarithmic potential $\ln z$ is the holomorphic primitive of the Cauchy kernel $\frac{1}{z}$, which is, up to a constant, the fundamental solution of the Cauchy-Riemann operator. In its turn this Cauchy kernel is the holomorphic primitive of the holomorphic function $-\frac{1}{z^{2}}$. At the other side, and still restricting to the upper half plane, the logarithmic potential $\ln z$ shows the holomorphic primitive $z(\ln z-1)$, which in its turn has a holomorphic potential, etc. Our aim is to construct, in the upper half of Euclidean space, a similar chain of monogenic potentials. Monogenicity is the central notion in Clifford analysis; it generalizes to higher dimension the notion of holomorphy in the complex plane, and is expressed by means of the generalized Cauchy-Riemann operator which is a combination of the derivative with respect to one of the variables, say $x_{0}$, and the so-called Dirac operator in the remaining variables $\left(x_{1}, \ldots, x_{n}\right)$. The Cauchy-Riemann operator and its conjugate linearize the Laplace operator whence Clifford analysis is entitled to be called a refinement of harmonic analysis. Starting point of our construction then is the fundamental solution of the Cauchy-Riemann operator, also called Cauchy kernel, and its relation to the Poisson kernel and its conjugate harmonic in upper half space. We then proceed by induction in both directions, downstream by differentiation, and upstream by primitivation, yielding an infinite chain of monogenic, and thus harmonic, potentials. Identifying the boundary of the upper half space with $\mathbb{R}^{m} \cong\left\{\left(x_{0}, \underline{x}\right) \in \mathbb{R}^{m+1}: x_{0}=0\right\}$, the distributional limits for $x_{0} \rightarrow 0+$ are computed; they divide into two classes which are linked by the Hilbert transform and encompass well-known distributions in $\mathbb{R}^{m}$ such as the Dirac distribution, the Hilbert kernel, the fundamental solutions of the Dirac and the Laplace operator, the square root of the negative Laplacian, and the like. It is also shown how each of the monogenic potentials constructed may be recovered by the convolution of an adjacent kernel in the chain with an appropriate boundary distribution.

\section{BASICS OF CLIFFORD ANALYSIS}

Clifford analysis (see e.g. [1, 2]) is a function theory which offers a natural generalization to higher dimension of holomorphic functions in the complex plane and refines harmonic analysis. Let $\left(e_{0}, e_{1}, \ldots, e_{m}\right)$ be the canonical orthonormal basis of Euclidean space $\mathbb{R}^{m+1}$, then the non-commutative multiplication in the real Clifford algebra $\mathbb{R}_{0, m+1}$ is governed by the rule $e_{\alpha} e_{\beta}+e_{\beta} e_{\alpha}=-2 \delta_{\alpha \beta}, \alpha, \beta=0,1, \ldots, m$, whence $\mathbb{R}_{0, m+1}$ is generated additively by the elements $e_{A}=e_{j_{1}} \ldots e_{j_{h}}$, where $A=\left\{j_{1}, \ldots, j_{h}\right\} \subset\{0, \ldots, m\}$, with $0 \leq j_{1}<j_{2}<\cdots<j_{h} \leq m$, and $e_{\emptyset}=1$. We identify the point $\left(x_{0}, x_{1}, \ldots, x_{m}\right) \in \mathbb{R}^{m+1}$ with the vector variable $x=x_{0} e_{0}+x_{1} e_{1}+\cdots x_{m} e_{m}=x_{0} e_{0}+\underline{x}$ and the point $\left(x_{1}, \ldots, x_{m}\right) \in \mathbb{R}^{m}$ with the vector variable $\underline{x}$. At the heart of Clifford analysis lies the so-called Dirac operator $\partial=\partial_{x_{0}} e_{0}+\partial_{x_{1}} e_{1}+\cdots \partial_{x_{m}} e_{m}=\partial_{x_{0}} e_{0}+\partial_{\underline{x}}$, which squares to the negative Lapace operator: $\partial^{2}=-\Delta_{m+1}$ and $\partial_{x}^{2}=-\Delta_{m}$. We also introduce the generalized Cauchy-Riemann operator $D=\overline{e_{0}} \partial=\partial_{x_{0}}+\overline{e_{0}} \partial_{\underline{x}}$ and its conjugate $\bar{D}=\overline{\partial_{x_{0}}}-\overline{e_{0}} \partial_{\underline{x}}$, 
which also decompose the Laplace operator: $D \bar{D}=\bar{D} D=\Delta_{m+1}$. We call a continuously differentiable function $F(x)$ in an open region $\Omega \subset \mathbb{R}^{m+1}$ (left-)monogenic if it satisfies $D F=0$ in $\Omega$, which is equivalent with $\partial F=0$. Singling out the basis vector $e_{0}$, we can decompose the real Clifford algebra $\mathbb{R}_{0, m+1}$ in terms of the Clifford algebra $\mathbb{R}_{0, m}$ as $\mathbb{R}_{0, m+1}=\mathbb{R}_{0, m} \oplus \overline{e_{0}} \mathbb{R}_{0, m}$. Similarly we decompose the functions considered as $F\left(x_{0}, \underline{x}\right)=F_{1}\left(x_{0}, \underline{x}\right)+\overline{e_{0}} F_{2}\left(x_{0}, \underline{x}\right)$ where $F_{1}$ and $F_{2}$ take their values in the Clifford algebra $\mathbb{R}_{0, m}$; mimicing functions of a complex variable, we will call $F_{1}$ the real part and $F_{2}$ the imaginary part of the function $F$.

\section{A CONJUGATE HARMONIC TO GREEN'S FUNCTION}

The notion of conjugate harmonics in the complex plane was generalized to higher dimension in [3] as follows. Given a harmonic function $U(x)$ in the upper half space $U H S=\left\{x \in \mathbb{R}^{m+1}: x_{0}>0\right\}$, there exists a harmonic function $V(x)$ in $U H S$ such that $F(x)=U(x)+\overline{e_{0}} V(x)$ is monogenic in $U H S$. This conjugate harmonic $V(x)$ and the corresponding monogenic function $F(x)$ may be derived from a harmonic potential $\widehat{H}(x)$ :

$$
V(x)=-\partial_{\underline{x}} \widehat{H}(x) \quad \text { and } \quad F(x)=\bar{D} \widehat{H}
$$

The fundamental solution of the Laplace operator $\Delta_{m+1}$ in $\mathbb{R}^{m+1}$, sometimes called Green's function, is given by

$$
K(x)=-\frac{1}{m-1} \frac{1}{a_{m}} \frac{1}{|x|^{m}}
$$

with $a_{m}$ the area of the unit sphere $S^{m-1}$ in $\mathbb{R}^{m}$; it thus satisfies, in distributional sense, $\Delta_{m+1} K(x)=\delta(x)$, where $\delta(x)$ stands for the Dirac distribution in $\mathbb{R}^{m+1}$. We consider it as a harmonic function in $U H S$ and construct its conjugate harmonic $L(x)$ by solving the following generalized Cauchy-Riemann system expressing the monogenicity of the function $K(x)+\overline{e_{0}} L(x)$ in $U H S$ :

$$
\begin{aligned}
& \partial_{x_{0}} K+\partial_{\underline{x}} L=0 \\
& \partial_{x_{0}} L+\partial_{\underline{x}} K=0
\end{aligned}
$$

Introducing the one variable function $F_{m}$ by

$$
F_{m}(v)=\int_{0}^{v} \frac{\eta^{m-1}}{\left(1+\eta^{2}\right)^{(m+1) / 2}} d \eta=\frac{v^{m}}{m}{ }_{2} F_{1}\left(\frac{m}{2}, \frac{m+1}{2} ; \frac{m}{2}+1 ;-v^{2}\right)
$$

with ${ }_{2} F_{1}$ a hypergeometric function, this conjugate harmonic $L(x)$ turns out to be

$$
L\left(x_{0}, \underline{x}\right)=\frac{1}{a_{m+1}} \frac{\underline{x}}{|\underline{x}|^{m}} F_{m}\left(\frac{|\underline{x}|}{x_{0}}\right)
$$

which, nota bene, is well defined for $\underline{x}=0$ since $\lim _{\underline{x} \rightarrow 0} L\left(x_{0}, \underline{x}\right)=0, x_{0}>0$. An alternative expression for this conjugate harmonic is obtained by introducing the one variable function $\widetilde{F_{m}}$ by

$$
\widetilde{F_{m}}(u)=\int_{0}^{u} \frac{d \xi}{\left(1+\xi^{2}\right)^{(m+1) / 2}}=u_{2} F_{1}\left(\frac{1}{2}, \frac{m+1}{2} ; \frac{3}{2} ;-u^{2}\right)
$$

leading to

$$
L\left(x_{0}, \underline{x}\right)=\frac{1}{a_{m+1}} \frac{\underline{x}}{|\underline{x}|^{m}}\left(\frac{\sqrt{\pi}}{2} \frac{\Gamma\left(\frac{m}{2}\right)}{\Gamma\left(\frac{m+1}{2}\right)}-\widetilde{F_{m}}\left(\frac{x_{0}}{|\underline{x}|}\right)\right)
$$

From this alternative expression it follows that for $\underline{x} \neq 0$ :

$$
l(\underline{x})=\lim _{x_{0} \rightarrow 0+} L\left(x_{0}, \underline{x}\right)=\frac{1}{2} \frac{1}{a_{m}} \frac{\underline{x}}{|\underline{x}|^{m}}
$$

in which we recognize, quite surprisingly, the fundamental solution of the Dirac operator $\partial_{\underline{x}}$ in $\mathbb{R}^{m}$. Note, by the way, that

$$
k(\underline{x})=\lim _{x_{0} \rightarrow 0+} K\left(x_{0}, \underline{x}\right)=-\frac{1}{m-1} \frac{1}{a_{m+1}} \mathrm{Fp} \frac{1}{|\underline{x}|^{m-1}}
$$


with Fp the so-called finite part distribution on the real line. This is, up to a multiplicative constant, the pseudodifferential operator $(-\Delta)^{-\frac{1}{2}}$ (see e.g. [4]). Moreover these two distributional limits form a Hilbert pair: $\mathscr{H}[k]=H * k=l$ and $\mathscr{H}[l]=H * l=k$, see the next section for the definition of the Hilbert transform and its kernel.

\section{DOWNSTREAM POTENTIALS}

As is well-known the Cauchy kernel $C\left(x_{0}, \underline{x}\right)$, i.e. the fundamental solution of the Dirac operator $\partial$, may be decomposed in $U H S$ in terms of the Poisson kernels:

$$
C(x)=\frac{1}{2} P(x)+\frac{1}{2} Q(x)
$$

where for $x_{0}>0$

$$
P\left(x_{0}, \underline{x}\right)=2 \partial_{x_{0}} K=-2 \partial_{\underline{x}} L=\frac{2}{a_{m}} \frac{x_{0}}{|x|^{m+1}} \quad \text { and } \quad Q\left(x_{0}, \underline{x}\right)=2 \partial_{x_{0}} L=-2 \partial_{\underline{x}} K=-\frac{2}{a_{m}} \frac{\underline{x}}{|x|^{m+1}}
$$

showing the following distributional limits for $x_{0} \rightarrow 0+$

$$
\lim _{x_{0} \rightarrow 0+} P\left(x_{0}, \underline{x}\right)=\delta(\underline{x}) \quad \text { and } \quad \lim _{x_{0} \rightarrow 0+} Q\left(x_{0}, \underline{x}\right)=H(\underline{x})
$$

the distribution

$$
H(\underline{x})=-\frac{2}{a_{m}} \operatorname{Pv} \frac{\underline{x}}{|\underline{x}|^{m+1}}
$$

being the convolution kernel of the Hilbert transform in $\mathbb{R}^{m}$. Both distributional limits are linked by the Hilbert transform $\mathscr{H}$ since, quite trivially $\mathscr{H}[\delta]=H * \delta=H$, and also $\mathscr{H}[H]=H * H=\delta$ in view of the involutive character of the Hilbert transform: $\mathscr{H}^{2}=\mathbf{1}$.

Seen the monogenicity of $C(x)$ in $U H S$ there holds $\bar{D} C=2 \partial_{x_{0}} C=-2 \overline{e_{0}} \partial_{\underline{x}} C=\bar{D} P=\bar{D}\left(\overline{e_{0}} Q\right)$ and we put

$$
C_{-2}=\bar{D} C=\frac{1}{2} A_{-2}+\frac{1}{2} \overline{e_{0}} B_{-2}
$$

It then follows that $C(x)$ is a monogenic potential of $C_{-2}(x)$, while $P(x)$ and $Q(x)$ are its conjugate harmonic potentials. Moreover $C_{-2}$ shows the following distributional limits for $x_{0} \rightarrow 0+$ :

$$
\frac{1}{2} a_{-2}=\lim _{x_{0} \rightarrow 0+} \frac{1}{2} A_{-2}=\frac{2}{a_{m+1}} \text { Fp } \frac{1}{|\underline{x}|^{m+1}} \quad \text { and } \quad \frac{1}{2} b_{-2}=\lim _{x_{0} \rightarrow 0+} \frac{1}{2} B_{-2}=-\partial_{\underline{x}} \delta
$$

Note that $\frac{1}{2} a_{-2}$ is nothing else but the convolution operator $-\partial_{\underline{x}} H=-H \partial_{\underline{x}}$, known as the Hilbert-Dirac operator (see [5]), or perhaps better known as the pseudodifferential operator square root of the negative Laplacian $(-\Delta)^{\frac{1}{2}}$. Also note that, again, both distributional limits form a Hilbert pair: $\mathscr{H}\left[\frac{1}{2} a_{-2}\right]=\frac{1}{2} b_{-2}$ and $\mathscr{H}\left[\frac{1}{2} b_{-2}\right]=\frac{1}{2} a_{-2}$. A direct and elegant way of proving this is to express the Hilbert transform as a convolution with the Hilbert kernel $H(\underline{x})$ and to revert to the thorough study, and in particular the results about convolution, of higher dimensional distributions in the Clifford analysis setting (see [6]).

Proceeding in the same way we can recursively define in $U H S$ the monogenic and conjugate harmonic potential kernels

$$
C_{-k-1}=\bar{D} C_{-k}=\bar{D}^{k} C=\frac{1}{2} A_{-k-1}+\frac{1}{2} \overline{e_{0}} B_{-k-1}, \quad k=1,2, \ldots
$$

Seen their monogenicity in $U H S$ there also holds that

$$
C_{-k-1}=2 \partial_{x_{0}} C_{-k}=\left(-2 \overline{e_{0}} \partial_{\underline{x}}\right) C_{-k}=\left(2 \partial_{x_{0}}\right)^{k} C=\left(-2 \overline{e_{0}} \partial_{\underline{x}}\right)^{k} C
$$

The real parts $\frac{1}{2} A_{-k-1}$ and imaginary parts $\frac{1}{2} B_{-k-1}$ of these kernels show distributional limits for $x_{0} \rightarrow 0+$ which are natural powers of the Dirac operator $\partial_{\underline{x}}$ acting on the Dirac distribution $\delta(\underline{x})$ or the Hilbert kernel $H(\underline{x})$ depending on the parity of $k$ :

(i) $\frac{1}{2} a_{-2 l}(\underline{x})=\lim _{x_{0} \rightarrow 0+} \frac{1}{2} A_{-2 l}\left(x_{0}, \underline{x}\right)=-2^{2 l-2} \partial_{\underline{x}}^{2 l-1} H$ 
(ii) $\frac{1}{2} b_{-2 l}(\underline{x})=\lim _{x_{0} \rightarrow 0+} \frac{1}{2} B_{-2 l}\left(x_{0}, \underline{x}\right)=-2^{2 l-2} \partial_{\underline{x}}^{2 l-1} \delta$

(iii) $\frac{1}{2} a_{-2 l-1}(\underline{x})=\lim _{x_{0} \rightarrow 0+} \frac{1}{2} A_{-2 l-1}\left(x_{0}, \underline{x}\right)=2^{2 l-1} \partial_{\underline{x}}^{2 l} \delta=2^{2 l-1}(-\Delta)^{l} \delta$

(iv) $\frac{1}{2} b_{-2 l-1}(\underline{x})=\lim _{x_{0} \rightarrow 0+} \frac{1}{2} B_{-2 l-1}\left(x_{0}, \underline{x}\right)=2^{2 l-1} \partial_{\underline{x}}^{2 l} H=2^{2 l-1}(-\Delta)^{l} H$

Moreover they form, once again, Hilbert pairs: $\mathscr{H}\left[\frac{1}{2} a_{-2 l}\right]=\frac{1}{2} b_{-2 l}$ and $\mathscr{H}\left[\frac{1}{2} a_{-2 l-1}\right]=\frac{1}{2} b_{-2 l-1}$.

\section{UPSTREAM POTENTIALS}

From the above mentioned relations between the Poisson kernels and the Green's function and its conjugate harmonic, it follows that $\bar{D} K=\bar{D}\left(\overline{e_{0}} L\right)=C$, which means that $K$ and $\overline{e_{0}} L$ are conjugate harmonic potentials and that

$$
C_{0}=\frac{1}{2} K+\frac{1}{2} \overline{e_{0}} L
$$

is a monogenic potential of the Cauchy kernel $C$ in $U H S$, thus satisfying

$$
\bar{D} C_{0}=2 \partial_{x_{0}} C_{0}=-2 \overline{e_{0}} \partial_{\underline{x}} C_{0}=C
$$

We also mentioned above that the boundary distributions $k(\underline{x})$ and $l(\underline{x})$ in $\mathbb{R}^{m}$ form a Hilbert pair. Moreover they are Dirac primitives of the distributional limits of the Poisson kernels: $\partial_{\underline{x}} k=-\frac{1}{2} H$ and $\partial_{\underline{x}} l=-\frac{1}{2} \delta$.

In order to construct harmonic and monogenic potentials for $C_{0}$ in $U H S$ we put $A_{1}=K * k=L * l$ and $B_{1}=K * l=$ $L * k$ and we verify that:

(i) $\partial_{x_{0}} A_{1}=\partial_{x_{0}} K * k=\frac{1}{2} P * k=\frac{1}{2} K$

(ii) $-\partial_{\underline{\underline{x}}} A_{1}=-\partial_{\underline{x}} K * k=\frac{1}{2} Q * k=\frac{1}{2} L$

(iii) $\partial_{x_{0}} B_{1}=\partial_{x_{0}} K * l=\frac{1}{2} P * l=\frac{1}{2} L$

(iv) $-\partial_{\underline{x}} B_{1}=-\partial_{\underline{x}} K * l=\frac{1}{2} Q * l=\frac{1}{2} K$

which are precisely the relations needed for $A_{1}$ and $B_{1}$ to be conjugate harmonic potentials in $U H S$ of $C_{0}$. It then follows that

$$
C_{1}=\frac{1}{2} A_{1}+\frac{1}{2} \overline{e_{0}} B_{1}
$$

is a monogenic potential in $U H S$ of $C_{0}$, and we have

$$
\bar{D} C_{1}=2 \partial_{x_{0}} C_{1}=-2 \overline{e_{0}} \partial_{\underline{x}} C_{1}=C_{0}
$$

The distributional limits of these harmonic potentials are given by

$$
\frac{1}{2} a_{1}=\lim _{x_{0} \rightarrow 0+} \frac{1}{2} A_{1}\left(x_{0}, \underline{x}\right)=\frac{1}{2} k * k(\underline{x})=\frac{1}{2} l * l(\underline{x})=\frac{1}{8} \frac{1}{a_{m}} \frac{1}{m-2} \frac{1}{|\underline{x}|^{m-2}}
$$

and

$$
\frac{1}{2} b_{1}=\lim _{x_{0} \rightarrow 0+} \frac{1}{2} B_{1}\left(x_{0}, \underline{x}\right)=\frac{1}{2} k * l(\underline{x})=\frac{1}{2} l * k(\underline{x})=-\frac{1}{4} \frac{1}{a_{m+1}} \frac{1}{m-1} \frac{\underline{x}}{|\underline{x}|^{m-1}}
$$

and again they form a Hilbert pair: $\mathscr{H}\left[a_{1}\right]=H * a_{1}=b_{1}$ and $\mathscr{H}\left[b_{1}\right]=H * b_{1}=a_{1}$. Note that in $a_{1}$ we recognize, up to a constant, the fundamental solution of the Laplace operator in $\mathbb{R}^{m}$. Moreover we can compute the explicit form of the potentials $A_{1}$ and $B_{1}$ making use again of the hypergeometric function $F_{m}$ introduced in Section 3:

$$
A_{1}\left(x_{0}, \underline{x}\right)=\frac{1}{2} \frac{1}{m-1} \frac{1}{a_{m+1}} \frac{1}{|\underline{x}|^{m-2}} F_{m-2}\left(\frac{|\underline{x}|}{x_{0}}\right)
$$

and

$$
B_{1}\left(x_{0}, \underline{x}\right)=\frac{1}{2} \frac{1}{a_{m+1}} \frac{x_{0} \underline{x}}{|\underline{x}|^{m}} F_{m}\left(\frac{|\underline{x}|}{x_{0}}\right)-\frac{1}{2} \frac{1}{a_{m+1}} \frac{1}{m-1} \frac{\underline{x}}{|\underline{x}|^{m-1}}
$$

showing that they are wel-defined for $\underline{x}=0$ and confirming the distributional limits for $x_{0} \rightarrow 0+$ mentioned above. 


\section{REFERENCES}

1. F. Brackx, R. Delanghe, F. Sommen, Clifford Analysis, Pitman Publishers (Boston-London-Melbourne, 1982).

2. R. Delanghe, F. Sommen, V. Souček, Clifford algebra and spinor-valued functions - A function theory for the Dirac operator, Kluwer Academic Publishers (Dordrecht, 1992).

3. F. Brackx, R. Delanghe, F. Sommen, On Conjugate Harmonic Functions in Euclidean Space

4. S. Helgason, Groups and Geometric Analysis, Pure and Applied Mathematics Academic Press (Orlando-London, 1984).

5. F. Brackx, H. De Schepper, Hilbert-Dirac Operators in Clifford Analysis, Chin. Ann. Math. 26B(1), 2005, 1-14

6. F. Brackx, B. de Knock, H. De Schepper, D. Eelbode, A Calculus Scheme for Clifford Distributions, Tokyo J. Math. 29(2), 2006, 495-513. 